\title{
Paracompactness in ordered spaces
}

by

R. Engelking (Warszawa) and D. Lutzer (Pittsburgh, Pa.)

Abstract. In this paper we prove that a generalized ordered space is not paracompact if and only if one of its closed subspaces is homeomorphic to a stationary subset of some ordinal having uncountable cofinality. We use that characterization to give easier proofs of two known results and to obtain two new theorems. We prove that a generalized ordered space $X$ is paracompact if it satisfies any of the following: (A) $X$ is perfectly normal; (B) $X$ has a compatible complete uniformity; (C) $X$ is $c$-semistratifiable in the sense of Martin; (D) $X$ has a $\mathscr{G}$-Souslin diagonal. Certain relevant examples are also presented.

1. Introduction. In this paper we present a new theory of paracompactness in generalized ordered spaces based on the notion of a stationary set of ordinals. A generalized ordered space is a triple $(X, \mathscr{T},<)$ where $<$ is a linear ordering of the set $X$ and where $\mathscr{T}$ is a topology on $X$ that is $T_{1}$ and has a base consisting of open sets which are order-convex $\left({ }^{1}\right)$. Obviously any linearly ordered topological space (abbreviated LOTS), i.e., a linearly ordered set equipped with its usual openinterval topology, is a generalized ordered space, as is any subspace of a LOTS when endowed with its relative topology, and one easily verifies that the familiar spaces known today as the Sorgenfrey line and the Michael line (see [S] and [M]) are also generalized ordered spaces. Our characterization of paracompactness in generalized ordered spaces hinges on the notion of a stationary set of ordinals. Let $\Omega$ be a limit ordinal. A subset $S$ of $\Omega$ is stationary in $\Omega$ if for each cofinal closed subset $\left(^{2}\right) C$ of $\Omega, S \cap C \neq \varnothing$. Obviously, if the cofinality of $\Omega$ is uncountable and if a subset $S$ of $\Omega$ contains a closed cofinal set, then $S$ is stationary in $\Omega$. It is known, however, that there are much more complicated stationary sets: for example, $\omega_{1}$ contains disjoint stationary subsets [R]. Nevertheless, the technical lemmas which we present later in this section will show that stationary sets in large ordinals have many features in common with closed cofinal sets in large ordinals.

(I) A subset $C$ of a linearly ordered set $(X,<)$ is order convex (or simply convex) provided $\{x \in X: a \leqslant x \leqslant b\} \subset C$ whenever $a, b \in C$.

${ }^{2}$ ) I.c., closed with respect to the usual order topology of $\Omega$; equivalently, if $\emptyset \neq D \subset C$ then $\sup _{\Omega}(D) \in C$.

4-Fundamenta Mathematicae t. XCrv 
Our characterization theorem, given in Section 2, asserts that a generalized ordered space $X$ is not paracompact if and only if some closed subspace of $X$ is homeomorphic to a stationary subset of an ordinal that has uncountable cofinality. Using that characterization we give new (and simplified) proofs of two known results and we obtain two new theorems related to recent work of Heath [He] and Braude [B]. We prove that a generalized ordered space $X$ is paracompact if it satisfies any of the following:

A. $X$ is perfectly normal $\left[\mathrm{L}_{2}\right]$;

B. $X$ has a compatible complete uniformity [I];

C. $X$ is $c$-semistratifiable in the sense of Martin [Ma];

D. $X$ has a $\mathscr{G}$-Souslin diagonal.

(Under hypotheses A, C and D we actually obtain hereditary paracompactness of $X$.)

The following notations appear frequently in subsequent sections. If $(X,<)$ is a linearly ordered set and $x \in X$, then $[x, \rightarrow[=\{y \in X: y \geqslant x\}$ and $] x, \rightarrow[$ $=\{y \in X: y>x\}$. Intervals such as $] x, y[,[x, y[$ and $[x, y]$ are analogously defined. If $T \subset X$ is not cofinal in $X$ we shall write $\sup _{X}(T)$ to denote the supremum of $T$ in $X$; usually the set $X$ will be order-complete so that there will be no question about the existence of the indicated supremum. If $\mathscr{C}$ is a collection of subsets of a set $X$ and if $p \in X$, then $\operatorname{St}(p, \mathscr{C})=\bigcup\{C \in \mathscr{C}: p \in C\}$. All of our spaces are assumed to be at least Hausdorff. Our topological terminology will conform to that of $[E]$ and terminology relating to ordinals (and not defined here) will follow [ $\mathrm{Ju}$ ].

The authors wish to thank R. Pol for suggestions which substantially shortened several of the proofs given in the original version of this paper.

We now present certain technical lemmas which are used throughout the paper.

1.1. LEMMA. Let $S$ be a stationary subset of an ordinal $\Omega$ having $\operatorname{cf}(\Omega)>\omega_{0}$ and let $T \subset S$ be a relatively closed cofinal subset of $S$. Then $T$ is also stationary in $\Omega$. In particular, the set of all non-isolated points of the subspace $S$ is stationary.

Proof. Supposing $T$ is not stationary in $\Omega$, let $C$ be a closed cofinal subset of $\Omega$ which is disjoint from $T$. Since $T$ is relatively closed in $S$ there is a closed subset $D$ of $\Omega$ having $T=S \cap D$. But then $C \cap D$ is a closed cofinal subset of $\Omega$ which is disjoint from $S$, and that is impossible. The second assertion of the lemma is now immediate.

Our next result is closely related to the classical "Pressing Down Lemma", a proof of which may be found in [Ju].

1.2. LEMMA. Let $\Omega$ be an ordinal having $\operatorname{cf}(\Omega)>\omega_{0}$ and suppose $S$ is a stationary subset of $\Omega$. For each $s \in S$ let $I(s)$ be an open subset of $\Omega$ which contains $s$ and let $\mathscr{I}=\{I(s): s \in S\}$. Then there is a point $x \in S$ such that $[x, \rightarrow[\subset \operatorname{Si}(x, \mathscr{I})$.

Proof. Let $\Gamma=\operatorname{cf}(\Omega)$. Supposing the lemma is false, inductively construct points $x(\gamma), y(\gamma) \in S$ such that

(a) if $\gamma<\delta<\Gamma$ then $x(\gamma)<y(\gamma)<x(\delta)$;

(b) if $\gamma<\Gamma$ then $y(\gamma) \notin \mathrm{S} i(x(\gamma), \mathscr{I})$;

(c) $\{x(\gamma): \gamma<\Gamma\}$ is cofinal in $\Omega$.
Because $\operatorname{cf}(\Omega)>\omega_{0}$, the closed set $C=\operatorname{cl}_{\Omega}\{x(\gamma): \gamma<\Omega\} \cap \operatorname{cl}_{\Omega}\{y(\gamma): \gamma<\Gamma\}$ is cofinal in $\Omega$ and it follows from (a) and (b) that $C \cap S=\varnothing$. Since that is impossible, the proof is complete.

1.3. Lemma. If $S$ is a stationary subset of the ordinal $\Omega$ having $\operatorname{cf}(\Omega)>\omega_{0}$ and if $S=\bigcup_{n=1}^{\infty} S(n)$, then one of the sets $S(n)$ is stationary in $\Omega$.

Proof. Let us first observe that, by countable compactness of $\Omega$, any countable intersection of closed cofinal subsets of $\Omega$ is nonvoid and hence cofinal in $\Omega$. To complete the proof, observe that if for each $n \geqslant 1 C(n)$ were a closed cofinal subset of having $C(n) \cap S(n)=\varnothing$ then $C=\bigcap_{n=1}^{\infty} C(n)$ would be a closed cofinal subset of $\Omega$ that is disjoint from $S$, and that is impossible.

We close this section with a result that gives yet another indication of the way in which stationary subsets of large ordinals behave like spaces of ordinals. The result is not needed in our paper and we state it without proof.

1.4. THEOREM. Let $S$ be a stationary subset of an ordinal $\Omega$ where $\operatorname{cf}(\Omega)>\omega_{0}$ and let $f$ be a continuous, real-valued function on $S$. Then $f$ is eventually constant, i.e., there is an $x \in S$ such that $f(x)=f(y)$ whenever $y \in S$ and $y \geqslant x$.

\section{Characterization of paracompact ordered spaces.}

2.1. LEMMA. Let $S$ be a stationary subset of an ordinal $\Omega$ where $\operatorname{cf}(\Omega)>\omega_{0}$. Then no (relatively) open cover of $S$ by bounded subsets of $\Omega$ can have a point-finite (relatively) open refinement.

Proof. Suppose that $\mathscr{U}$ is a relatively open cover of $S$ by bounded subsets of $\Omega$ and suppose that $\mathscr{U}$ is point-finite at each point of $S$. Write $\mathcal{U}=\{V(i) \cap S \mid i \in I\}$ where each $V(i)$ is a bounded open subset of $\Omega$ and apply (1.2) to obtain a contradiction.

Our next lemma is related to a result in $\left[\mathrm{L}_{4}\right]$.

2.2. LEMMA. If $X$ is a non-paracompact LOTS then some closed subspace of $X$ is homeomorphic to a stationary subset of an ordinal $\Omega$ where $\Omega=\operatorname{cf}(\Omega)>\omega_{0}$.

Proof. Let $X^{+}$denote the Dedekind completion of $X$. Since $X$ is not paraProof. Let $X^{+}$compact, some point $u \in X^{+} \backslash X$ has the property that $u$ is not a $Q$-gap of $X$, say
comote from below [GH], i.e., if $\Omega$ is the unique ordinal which is cofinal in $\{x \in X: x<u\}$ and which has $\operatorname{cf}(\Omega)=\Omega$, then $\Omega>\omega_{0}$ and for any strictly increasing net $\{x(\alpha): \alpha<\Omega\}$ in $X$ whose supremum (in $X^{+}$) is $u$, there is a limit ordinal $\lambda<\Omega$ such that $\sup \{x(\alpha) ; \alpha<\lambda\} \in X$, the supremum being taken in $X^{+}$. Fix any such net; we may assume that for any limit ordinal $\lambda<\Omega, \sup \{x(\alpha): \alpha<\lambda\}$ is either $x(\lambda)$ or else is in $X^{+} \backslash X$. Let $S=\{\lambda<\Omega: \lambda$ is a limit ordinal and $\sup \{x(\alpha): \alpha<\lambda\}=x(\lambda) \in X\}$. Then $S$ is stationary in $\Omega$ because if some cofinal closed set $C \subset \Omega$ were disjoint from $S$ then one could use the net $\{x(\alpha): \alpha \in C\}$ to show that $u$ is a $Q$-gap from 
below. Also, the function $f: S \rightarrow X$ given by $f(\lambda)=x(\lambda)$ for each $\lambda \in S$ is a homeomorphism of $S$ onto a closed subspace of $X$.

2.3. THEOREM. The following properties of a generalized ordered space $X$ are equivalent:

(a) $X$ is not paracompact;

(b) for some ordinal $\Omega$ with $\operatorname{cf}(\Omega)=\Omega>\omega_{0}$, some stationary subset of $\Omega$ is homeomorphic to a closed subspace of $X$;

(c) for some ordinal $\Omega$ with $\mathrm{cf}(\Omega)>\omega_{0}$, some stationary subset of $\Omega$ is homeomorphic to a closed subspace of $X$.

Proof. That (b) implies (c) is trivial and (c) implies (a) in the light of (2.1). In case $X$ is a LOTS then (2.2) applies to show that (a) implies (b). Now consider the case where $X$ is a generalized ordered space. Using the techniques of $\left[\mathrm{L}_{2}\right]$, we embed $X$ into a LOTS $X^{*}$ in such a way that every point of $X^{*} \backslash X$ is isolated in $X^{*}$. If $X$ is not paracompact, it follows that $X^{*}$ cannot be paracompact so that by the first part of the proof there is a stationary subspace $S$ of an ordinal $\Omega$ having $\operatorname{cf}(\Omega)$ $=\Omega>\omega_{0}$ and a homeomorphism $h$ of $S$ onto a closed subspace $Y$ of $X^{*}$. Let $T$ be the set of non-isolated points of the subspace $S$; then $T$ is stationary in $\Omega$ according to (1.1) and $h$ sends $T$ onto a closed subspace of $X^{*}$. Since all points of $X^{*} \backslash X$ are isolated in $X^{*}, h(T) \subset X$.

Since any subspace of a generalized ordered space is itself a generalized ordered space, our next result follows directly from (2.3).

2.4. THEOREM. The following properties of a generalized ordered space $X$ are equivalent:

(a) $X$ is not hereditarily paracompact;

(b) some subspace of $X$ is homeomorphic to a stationary subset of an ordinal $\Omega$ having $\operatorname{cf}(\Omega)=\Omega>\omega_{0}$

(c) some subspace of $X$ is homeomorphic to a stationary subset of an ordinal $\Omega$ having $\operatorname{cf}(\Omega)>\omega_{0}$.

2.5. Remark. The reader might conjecture that the conclusions of 2.3 and 2.4 could be strengthened to say that a non-paracompact generalized ordered space must contain a subspace homeomorphic to a closed cofinal subset of an ordinal having uncountable cofinality. That is not true, as the following example shows. Let $Z$ be the set of integers and let $L=\left\{\lambda<\omega_{2}: \lambda\right.$ is a limit ordinal and $\operatorname{cf}([0, \lambda[)$ $\left.=\omega_{0}\right\}$. Let

$$
X=\left\{(\alpha, n) \in \omega_{2} \times Z: \text { either } \alpha \in \omega_{2} \backslash L \text { and } n=0 \text { or else } \alpha \in L \text { and } n \leqslant 0\right\}
$$

and order $X$ lexicographically. Then $X$, with the open-interval topology, is a nonparacompact LOTS in which no countable set has a limit point. Thus no ordinal having uncountable cofinality can be topologically embedded in $X$. A more complicated example is described in $\left[\mathrm{L}_{4}\right]$ : a first-countable non-paracompact LOTS which has $s_{1}$ points and yet which contains no topological copy of $\omega_{1}$.

\section{Two known results.}

3.1. THEOREM $\left[\mathrm{L}_{2}\right]$. A perfectly normal generalized ordered space is paracompact.

Proof. By (2.3) it will be enough to show that if $\Omega$ is an ordinal with uncount able cofinality and if $S$ is a stationary subset of $\Omega$ then $S$ (with the relative topology) cannot be perfectly normal. To that end, let $T$ be the set of non-isolated points of $S$ and suppose that $T$ is a $G_{\delta}$-subset of $S$. Write $T=\bigcap_{n=1}^{\infty}(S \cap U(n))$ where each $U(n)$ is an open subset of $\Omega$. According to (1.1) there is a $t(n) \in T$ such that $\left[t(n), \rightarrow\left[\subset U(n)\right.\right.$. Since $\operatorname{cf}(\Omega)>\omega_{0}$ there is a point $t^{\prime} \in T \cap\left(\bigcap_{n=1}^{\infty}[t(n), \rightarrow[)\right.$. Then $\left[t^{\prime}, \rightarrow\left[\dot{\subset} U(n)\right.\right.$ for each $n \geqslant 1$ so that $S \cap\left[t^{\prime}, \rightarrow\left[\subset \bigcap_{n=1}^{\infty}(S \cap U(n))=T\right.\right.$. But that is impossible because if $t^{\prime \prime}$ is the first point of $S$ that is greater than $t^{\prime}$, then $\left\{t^{\prime \prime}\right\}$ is open in $S$ so that $t^{\prime \prime} \notin T$ even though $t^{\prime \prime} \in S \cap\left[t^{\prime}, \rightarrow[\right.$.

Let $\mathscr{U}$ be a uniformity for $X$ (i.e., a certain family of subsets of $X \times X$; see [E]) and let $\mathscr{T}$ be the associated topology for $X$. A filterbase $\mathscr{F}$ is said to be Cauchy with respect to $\mathscr{U}$ if for each $U \in \mathscr{U}$ some $F \in \mathscr{F}$ has $F \times F \subset U$, and the uniformity $\mathscr{U}$ is said to be complete if every filterbase $\mathscr{F}$ of $\mathscr{T}$-closed sets which is Cauchy with respect to $\mathscr{U}$ has $\cap \mathscr{F} \neq \varnothing$. Finally, a space $X$ is Dieudonné complete if some complete uniformity on $X$ induces the topology of $X$. Our next theorem was obtained for LOTS by Ishii [I].

3.2. THEOREM. If a generalized ordered space is Dieudonné complete then it is paracompact.

Proof. According to (2.3) it will be enough to show that if $S$ is a stationary subset of an ordinal $\Omega$ with $\operatorname{cf}(\Omega)>\omega_{0}$, then $S$ cannot have a compatible complete uniformity. Let $\mathcal{U}$ be any uniformity for $S$ and let $U \in \mathscr{U}$. For each $x \in S$ let $I(x)$ be an open convex subset of $\Omega$ containing $x$ and having $(I(x) \cap S) \times(I(x) \cap S) \subset U$. According to (1.2) there is a point $x(U) \in S$ such that, with $\mathscr{I}=\{I(x): x \in S\}$, $[x(U), \rightarrow[\subset \mathrm{St}(x(U), \mathscr{I})$. Therefore, since members of $\mathscr{I}$ are convex, $(y, z) \in U$ whenever $y, z \in S \cap[x(U), \rightarrow[$ so that the collection $\mathscr{F}=\{S \cap[x, \rightarrow[: x \in S\}$ is seen to be a filterbase of closed sets which is Cauchy with respect to $\mathscr{U}$ and yet which has $\cap \mathscr{F}=\varnothing$. Thus $S$ cannot be Dieudonné complete.

3.3. Remark. It is clear that the proof of (3.1) actually shows that a perfectly normal generalized ordered space is hereditarily paracompact, in the light of (2.4). That fact also follows from general theory since any paracompact space in which every closed set is a $G_{\delta}$ is hereditarily paracompact. In fact, an even stronger result is true for generalized ordered spaces: as noted in $\left[\mathrm{L}_{4}\right]$, any paracompact generalized ordered space in which each point is a $G_{\delta}$ must be hereditarily paracompact. Of course, there is no hope that every Dieudonné complete generalized ordered space must be hereditarily paracompact-consider the compact LOTS $\left[0, \omega_{1}\right]$. One easy consequence of (3.2) is that if a generalized ordered space is realcompact [E] then it is paracompact. That consequence may also be deduced from Theorem 1.4. 
4. Paracompactness of $c$-semistratifiable ordered spaces. In this section we present a generalization of a theorem announced by $\mathrm{R}$. W. Heath [He] asserting that a quasi-metrizable LOTS is paracompact. We begin by reminding the reader of some definitions. A quasi-metric on a set $X$ is a real-valued function $d$ on $X \times X$ such that for any $x, y, z$ in $X, d(x, y) \geqslant 0$ with $d(x, y)=0$ if and only if $x=y$ and such that $d(x, y) \leqslant d(x, z)+d(z, y)$. The associated quasi-metric topology on $X$ is the one for which $\{\{y \in X: d(x, y)<\varepsilon\}: \varepsilon>0\}$ is a neighborhood base at each point $x \in X$. The literature contains many definitions which are at least formally weaker than quasi-metrizability: the $\gamma$-spaces of Hodel, the co-Nagata spaces of Martin, the spaces which admit a co-convergent open neighborhood assignment introduced by Subella, and the spaces which have a compatible $o$-metric having property $\pi$ studied by Nedev and Choban. Lindgren and Fletcher [LF] proved that those four definitions are equivalent and it is easily seen that the following definition is equivalent to Hodel's definition of $\gamma$-spaces [Ho]: a space $X$ is a $\gamma$-space if there is a collection $\{g(n, x): n \geqslant 1, x \in X\}$, called a $\gamma$-structure for $X$, of open subsets of $X$ such that (a) for each $x \in X,\{g(n, x): n \geqslant 1\}$ is a neighborhood base for $x$, and (b) for each $x \in X$ and $n \geqslant 1$ there is an integer $m=m(x, n) \geqslant n$ such that if $y \in g(m, x)$ then $g(m, y) \subset g(n, x)$. It is also clear that such spaces are $c$-semistratifiable in the sense of Martin [Ma], i.e., each point $x$ of $X$ has a sequence $\{g(n, x): n \geqslant 1\}$ of open neighborhoods such that for any compact $C \subset X$, if $g(n, C)$ $=\bigcup\{g(n, x): x \in C\}$, then $\bigcap\{g(n, C): n \geqslant 1\}=C$; the collection $\{g(n, x) \mid n \geqslant 1$, $x \in X\}$ is called a $c$-semistratification of $X$. It is easily seen that a semistratifiable space [C] is $c$-semistratifiable.

4.1. THEOREM. A c-semistratifiable generalized ordered space is paracompact.

Proof. In the light of (2.3) it will be enough to show that if $S$ is a stationary subset of $\Omega$, where $\operatorname{cf}(\Omega)=\Omega>\omega_{0}$, then $S$ cannot be $c$-semistratifiable. Arguing indirectly, suppose $\{g(n, x): n \geqslant 1, x \in S\}$ is a $c$-semistratification of $S$. For each $x \in S$ let $G(n, x)$ be an open convex bounded subset of $\Omega$ having $x \in S \cap G(n, x)$ $\subset g(n, x)$ and $G(n+1, x) \subset G(n, x)$. Let $\mathscr{G}(n)=\{G(n, x): x \in S\}$. By (1.2) there are points $x(n) \in S$ such that $\left[x(n), \rightarrow\left[\subset \mathrm{S}(x(n), \mathscr{G}(n))\right.\right.$ and, since $\operatorname{cf}(\Omega)>\omega_{0}$, a point $y \in S$ such that $y \geqslant x(n)$ for every $n$. Bzcause the sets $G(n, x)$ are all convex, $[y, \rightarrow[\subset \mathrm{S}(y, \mathscr{G}(n))$ for every $n$.

Let $S(n)=\{s \in S: y \in G(n, s)\}$. Each set $S(n)$ is cofinal in $\Omega$ so that, $S$ being stationary, there is a point $s \in(S \cap] y, \rightarrow[) \cap\left(\cap\left\{\mathrm{cl}_{\Omega}(S(n)): n \geqslant 1\right\}\right)$ which is also a limit point of $S$ (see Lemma 1.1). Because $S$ is first-countable, there are points $s(n) \in S(n) \cap] y, \rightarrow[$ such that the sequence $\langle s(n)\rangle$ converges to $s$. But then the set $C=\{s\} \cup\{s(n): n \geqslant 1\}$ is compact and yet $y \in \bigcap\{G(n, C): n \geqslant 1\}$ even though $y \notin C$. That contradiction completes the proof.

Observe that the only compact sets considered in our proof were convergent sequences.

We next give two examples which show that our Theorem 4.1 is not a corollary of (3.1) and is more general that Heath's theorem on quasi-metrizable LOTS, mentioned above.
4.2. EXAMPLE. There is a quasi-metrizable LOTS which is not perfectly normal. Let $R, Q$ and $Z$ denote respectively the sets of real numbers, rational numbers, and integers. Let $X=\{(x, n) \in R \times Z$ : if $x$ is rational then $n=0\}$ and order $X$ lexicographically. The open-interval topology of that order has a $\sigma$-disjoint base and is, therefore, quasi-metrizable [SZ].

Alternatively one can explicitly define a quasi-metric on $X$ by:

$$
d((x, n),(y, m))=\left\{\begin{array}{lll}
\frac{|x-y|}{1+|x-y|} & \text { if } & x \in Q, \\
1 & \text { if } & x \notin Q \text { and }(x, n) \neq(y, m), \\
0 & \text { if } & (x, n)=(y, m) .
\end{array}\right.
$$

However, $X$ is not perfectly normal: the set $\{(x, 0): x \in Q\}$ is easily seen to be a closed subset of $X$ which is not a $G_{\delta}$. (This example will be used again in Section 5.)

4.3. EXAMPLE. There is a $c$-semistratifiable generalized ordered space which is not a $\gamma$-space and is not, therefore, quasi-metrizable. With $R, P$ and $Q$ denoting, respectively, the sets of real, irrational and rational numbers, let $Y$ denote the space obtained when $R$ is retopologized by taking basic neighborhoods of a point $x$ to be intervals of the form $[x, x+\varepsilon[$ if $x \in P$ and of the form $] x-\varepsilon, x]$ if $x \in Q$. Then $Y$ is a generalized ordered space which is $c$-semistratifiable: for each $x$ and each $n \geqslant 1$, let $g(n, x)=] x-1 / n, x+1 / n[$. However, $Y$ is not a $\gamma$-space. For suppose that $\mathscr{H}=\{h(n, y): n \geqslant 1$ and $y \in Y\}$ is a $\gamma$-structure for $Y$ as defined above. Replacing each $h(n, y)$ by a smaller set if necessary, we may assume that each $h(n, y)$ is a basic neighborhood, as defined above, of the point $y$. For each $j, k \geqslant 1$ let $P(j, k)$ $=\{y \in P: m(y, 1)=j$ and $[y, y+1 / k[\subset h(j, y)\}$, where $m(y, 1)$ is the integer described in the definition of a $\gamma$-space. Because $P=\bigcup\{P(j, k): j, k \geqslant 1\}$, there are integers $J$ and $K$ and an open interval $] a, b[$ such that $] a, b\left[\subset \operatorname{cl}_{R}(P(J, K))\right.$, the indicated closure being taken with respect to the usual topology of $R$. Choose an $r \in Q \cap] a, b[$ and a point $y \in P(J, K)$ such that (i) $y \in h(J, r)$ and (ii) $|y-r|<1 / K$. It follows from (i) that $y<r$ and that some $z<y$ belongs to $h(J, r)$ because $h(J, r)$ is a basic neighborhood of $r$. From (ii) it follows that $r \in[y, y+1 / K[\subset h(J, y)$. But then our assumption that $\mathscr{H}$ is a $\gamma$-structure for $Y$ yields $z \in h(J, r) \subset h(1, y)$ $c[y, \rightarrow[$ and that contradiction completes the proof.

The previous example is a generalized ordered space but is not a LOTS. Indeed, our next theorem shows that if one considers only spaces with the open-interval topology of some linear ordering, then many of the concepts considered in this section reduce to the same thing. Since the theorem is not needed in our current paper we state it without proof.

4.4. THEOREM. Let $X$ be a LOTS. Then (1) $X$ is a c-semistratifiable if and only

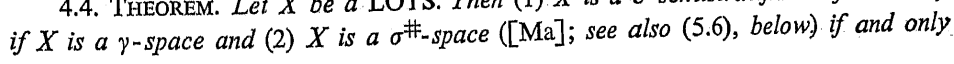


if $X$ is quasi-metrizable by a quasi-metric $d$ with the property that for any $x, y, z$ in $X, d(x, y) \leqslant \max \{d(x, z), d(z, y)\}$.

4.5. Remark. Since any subspace of a $c$-semistratifiable generalized ordered space is itself a $c$-semistratifiable generalized ordered space, it follows immediately that such a space is hereditarily paracompact.

5. Ordered spaces with $\mathscr{G}$-Souslin diagonals. Before giving the results of this section we need some notation. Let $\sigma$ be the set of all finite sequences of positive integers and let $\Sigma$ be the set of all infinite sequences of positive integers. For a point $\underline{n}=\left(n_{1}, n_{2}, \ldots\right)$ of $\Sigma$ and for $k \geqslant 1$, write $\underline{n} / k=\left(n_{1}, \ldots, n_{k}\right)$. A space $X$ is said to have a $\mathscr{G}$-S Juslin diagonal if for each $p \in \bar{\sigma}$ there is an open subset $G(p)$ of $X \times X$ such that $\{(x, x): x \in X\}=\bigcup\left\{\bigcap_{k=1}^{\infty} G(\underline{n} / k): \underline{n} \in \Sigma\right\}$. Braude [B] has proved that a compact Hausdorff spaçe having a $\mathscr{G}$.S suslin diagonal is metrizable, thereby generalizing certain theorenis of Šneľder. In view of the parallelism between the metrization theory of compact Hausdorff spaces and of LOTS $\left[\mathrm{L}_{3}\right]$, one might be led to the conjecture that a LOTS having a $\mathscr{G}$-S Juslin diagonal is metrizable. That conjecture would be false as our Example 5.2 shows. Before presenting the example we need a lemma.

5.1. Lemma. Suppose that each point of a space $X$ is a $G_{\delta}$ in $X$ and that $X$ has at most countably many non-isolated points. Then $X$ has a $\mathscr{G}$-Souslin diagonal.

Proof. Let $\mathscr{G}$ denote the family of all open subsets of $X \times X$. Since $\mathscr{G}$ is closed under the formation of finite unions, the family $\mathscr{A}$ obtained by applying the Souslin $A$-operation to $\mathscr{G}$ (i.e., the family of $\mathscr{G}$-Souslin sets) is closed under the formation of countable unions and countable intersections $([\mathrm{KM}]$ or $[\mathrm{Ch}])$. If $I$ is the set of isolated points of $X$, then the diagonal

$$
\Delta=\{(x, x): x \in I\} \cup(\bigcup\{\{(x, x)\}: x \in X \backslash I\})
$$

is a countable union of members of $\mathscr{A}$, so $\Delta \in \mathscr{A}$ as required.

5.2. EXAMPLE. There is a non-metrizable LOTS having a $\mathscr{G}$-Souslin diagonal; hence a LOTS can have a $\mathscr{G}$-Suuslin diagonal without having a $G_{\delta}$-diagonal. Let $X$ be the space constructed in Example 4.2. Then $X$ is not metrizable (and hence the diagonal of $X$ is not a $G_{\delta}$-set by $\left.\left[\mathrm{L}_{1}\right]\right)$ and yet $X$ is first-countable and paracompact. Further, the only non-isolated points of $X$ are points $(x, 0)$ where $x$ is rational; according to (5.1), $X$ has a $\mathscr{G}$-S Juslin diagonal. prove:

Paracompactness of the space in Example 5.2 is not accidental since we can

5.3. THĖOREM. A generalized ordered space having a $\mathscr{G}$-Souslin diagonal is paracompuct.
Proof. It will be enough to show that if $S$ is a stationary subset of an ordinal $\Omega$ having $\operatorname{cf}(\Omega)=\Omega>\omega_{0}$ then $S$ does not have a $\mathscr{G}$-Souslin diagonal. We argue indirectly, supposing that

$$
\Delta=\{(s, s): s \in S\}=\bigcup\left\{\bigcap_{k=1}^{\infty} G(\underline{n} / k): \underline{n} \in \Sigma\right\}
$$

where each $G(\underline{n} / k)$ is open in $S \times S$. We may assume that $G(\underline{n} / k+1) \subset G(\underline{n} / k)$ for each $k$. For each $x \in S$ let $\Delta(x)=\{(y, y) \in \Delta: y \geqslant x\}$. We assert

(*) there is an $\underline{n} \in \Sigma$ such that for each $k \geqslant 1, G(\underline{n} / k) \supset \Delta(x(k))$ for some $x(k) \in S$. Suppose that $(*)$ is false; then

(**) for each $\underline{n} \in \Sigma$ there is a $k(n) \geqslant 1$ such that $G(\underline{n} / k(n))$ contains no set $\Delta(x)$. The collection $\mathscr{G}^{\prime}=\{G(n / k(n)): n \in \Sigma\}$ is countable and $\Delta \subset \cup \mathscr{G}^{\prime}$ so that for some $n \in \Sigma$ the open set $T=\{x \in S:(x, x) \in G(n / k(n))\}$ must be stationary in $\Omega$ (see $1 . \overline{3})$. Hence, there is a point $p \in T$ such that $\bar{\Delta}(p) \subset G(\underline{n} / k(n))$, contrary to (**). Thus, (*) holds.

Take any $\underline{n}$ and any sequence $\langle x(k)\rangle$ satisfying (*). As in the proof of (3.2), there must be a point $y(k) \in S$ having $\left(\left[y(k), \rightarrow[)^{2} \subset G(\underline{n} / k)\right.\right.$. Since $\operatorname{cf}(\Omega)>\omega_{0}$, there are distinct points $u, v$ of $S$ both of which are greater than every $y(k)$. But then $(u, v) \in \bigcap_{k=1}^{\infty} G(n \mid k) \subset \Delta$ which is impossible. That contradiction completes the proof.

W. Lindgren has pointed out another proof of (5.3) which largely avoids working with the $\mathscr{G}$-Souslin diagonal structure and which reduces (5.3) to our earlier Theorem 4.1.

5.4. THEOREM. If a generalized ordered space has a $\mathscr{G}$-Souslin diagonal, then it is c-semistratifiable.

Proof. Suppose $\Delta=\bigcup\left\{\bigcap_{k=1}^{\infty} G(\underline{n} / k): \underline{n} \in \Sigma\right\}$ where each $G(\underline{n} / k)$ is open in $X \times X$. For each $\left(n_{1}, \ldots, n_{k}\right) \in \sigma$ let $\mathscr{H}\left(n_{1}, \ldots, n_{k}\right)=\{U \subset X: U$ is open and $U \times U$ $\left.\subset G\left(n_{1}, \ldots, n_{k}\right)\right\}$ and let $\Psi=\left\{\mathscr{H}\left(n_{1}, \ldots, n_{k}\right):\left(n_{1}, \ldots, n_{k}\right) \in \sigma\right\}$. Then $\Psi$ is a countable family of collections of open sets in $X$ with the property that given $x \neq y$ in $X$, some $\mathscr{H} \in \Psi$ has the property that $x \in \mathrm{S}(x, \mathscr{H}) \subset X \backslash\{y\}$. It is known [Sc] that any generalized ordered space is hereditarily orthocompact, where we say that a space $Y$ is orthocompact provided that for each open cover $\mathscr{U}$ of $Y$, there is an open cover $\mathscr{V}$ of $Y$ which refines $\mathscr{U}$ and has the property that for each $y \in Y$ the set $\bigcap\{V \in \mathscr{V}: y \in V\}$ is open. Therefore, for each $\mathscr{H} \in \Psi$ we may find an open collection $\mathscr{H}^{*}$ in $X$ which covers $\bigcup \mathscr{H}$, refines $\mathscr{H}$ and has the property that for each $y \in \bigcup \mathscr{H}$ the set $\bigcap\left\{V \in \mathscr{H}^{*}: y \in V\right\}$ is open. For each $\mathscr{H} \in \Psi$ let $\mathscr{F}(\mathscr{H})$ $=\left\{X \backslash V: V \in \mathscr{H}^{*}\right\}$. Then each $\mathscr{F}(\mathscr{H})$ is a closure preserving collection in $X$ and given $x \neq y$ in $X$ there is a set $K \in \bigcup\{\mathscr{F}(\mathscr{H}): \mathscr{H} \in \Psi\}$ such that $x \in K \subset X \backslash\{y\}$. 
(In the terminology of [Ma], $X$ is a $\sigma^{\#-\text { space.) }}$ But it is known [Ma] that every

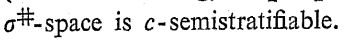

5.5. Remark. Since the spaces satisfying the hypotheses of Theorem 5.3 form a hereditary class, we see that a generalized ordered space with a $\mathscr{G}$-Souslin diagonal must be hereditarily paracompact. Furthermore, (5.4) shows that a generalized ordered space is $c$-semistratifiable (and hence paracompact) if it has a quasi- $G_{\delta}$ diagonal (i.e., if it admits a countable collection $\Psi$, as in the proof of (5.4)).

\section{References}

[B] E. J. Braúde, Compact $\mathscr{G}$-Souslin sets are $G_{\delta}$ 's, Proc. Amer. Math. Soc. 40 (1973), pp. $250-252$.

[C] G. Creede, Concerning semistratifiable spaces, Pacific J. Math. 32 (1970), pp. 47-54

[Ch] J. P. R. Christensen, Topology and Borel Structure, Mathematics Studies 10, Amsterdam 1974.

[E] R. Engelking, General Topology, Warszawa 1977.

[GH] L. Gillman and M. Henriksen, Concerning rings of continuous functions, Trans. Amer Muth. Soc. 77 (1954), pp. 340-362.

[He] R. W. Heath, Some new results on quasi-metric spaces, Topology Conference, Athens, Ohio, October, 1972 (oral communication).

[I] T. Ishii, A new characterization of paracompactness; Proc. Japan Acad. 35 (1959), pp. $435-436$.

[Ju] I. Juhasz, Cardinal Functions in Topology, Math. Centre Tracts 34, Math. Centrum, Amsterdam 1971

[KM] K. Kuratowski and A. Mostowski, Set Theory, Amsterdam 1968.

[LF] W. Lindgren and P. Fletcher, Locally quasi-uniform spaces with countable bases, Duke Math. J. 41 (1974), pp. 231-240.

$\left[\mathrm{L}_{1}\right] \quad$ D. Lutzer, A metrization theorem for linearly orderable spaces, Proc. Amer. Math. Soc. 22 (1969), pp. 557-558.

$\left[\mathrm{L}_{2}\right] \quad-$ On generalized ordered spaces, Dissertationes Math. 89 (1971).

$\left[\mathrm{L}_{*}\right]$ - On quasi-uniform bases, Proc. Oklahoma Topology Conference 1972, University of Oklahoma, Norman, Oklahoma, pp. 104-117.

$\left[\mathrm{L}_{4}\right]$ - Ordinals and paracompactness in ordered spaces, TOPO 72, Proceedings of the Second Pittsburgh Topology Conference, Lecture Notes in Mathematics 378, pp. 258-266.

[M] E. Michael, The product of a normal space and a metric space need not be normal, Bull. Amer. Math. Soc. 69 (1963), pp. 375-376.

[Ma] H. Martin, Topological spaces in which compact sets are uniformly $G_{\delta}$, preprint

[R] M. E. Rudin, A subset of the countable ordinals, Amer. Math. Monthly, 64 (1957), p. 351.

[S] R. H. Sorgenfrey, On the topological product of paracompact spaces, Bull. Amer. Math. Soc. 53 (1947), pp. 631-632.

[Sc] B. Scott, Toward a product theory for orthocompactness, preprint

[SZ] M. Sion and G. Zelmer, On quasi-metrizability, Canad. J. Math. 19 (1967), pp. 1243-1249,

\section{DEPARTMENT OF MATHEMATICS AND MECHANICS}

WARSAW UNIVERSITY

Warsaw, Poland

DEPARTMENT OF MATHEMATIC

UNIVERSITY OF PITTSBURGH

Pittsburgh, P.a.

Accepté par la Rédaction le 9. 1. 1975

\section{Homogeneity, universality and saturatedness of limit reduced powers (II)}

by

B. Weglorz (Wrocław)

Abstract. We give some necessary conditions on the pair $\mathscr{D}, \mathscr{G}$ ), where $\mathscr{D}$ is an ultrafilter on $I$ and $\mathscr{G}$ is a filter on $I \times I$, which imply that for every structure $\mathfrak{A}$, the limit ulfrapower $\mathfrak{A}_{\mathscr{D}}^{I} \mid \mathscr{G}$ is $x$-universal (or $x$-saturated)

The paper is a continuation of [5]. In $\S 1$, we prove Embedding Theorem which says that every limit ultrapower $\mathfrak{U}_{\mathscr{D}}^{I} \mid \mathscr{G}$ contains a lot of elementary submodels which are isomorphic to ultrapowers of $\mathfrak{A}$ reduced by ultrafilters which are obtained in a natural way from $\mathscr{D}$. The idea of Embedding Theorem (in fact contained in the proof of Theorem 4 in [4]) was suggested to the author by the proof of Wierzejewski's Theorem 1 in [5].

In $\S 2$, we apply Embedding Theorem to give some necessary combinatorial conditions on the pair $(\mathscr{D}, \mathscr{G})$ which imply that for every structure $\mathfrak{A}$, the limit ultrapower $\mathfrak{A}_{\mathscr{D}}^{T} \mid \mathscr{G}$ is $x$-universal (or $x$-saturated).

We assume that the reader is familiar with the notion and basic properties of limit reduced powers (see [1]). We also assume the familiarity with the notions of $(x, \omega)$-regular and $x$-good filters (see e.g. [2]). The only non standard notation is the following: if $\varrho$ is an equivalence relation on $I$ then by $I / \varrho$ we denote the set of all $\varrho$-equivalence classes over $I$. We write $I / \varrho=\left\{I_{j}: j \in J\right\}$ to denote that $I_{j}$ 's are all the $\varrho$-equivalence classes of elements of $I$.

The author is deeply indebted to L. Pacholski and J. Wierzejewski for a lot of very stimulating discussions which helped to formulate and prove the results presented below.

$\S$ 1. Embedding Theorem. Let $\mathscr{D}$ be a filter on $I$ and $\varrho$ an equivalence relation on $I$. Let $I / \varrho=\left\{I_{j}: j \in J\right\}$. The family $\mathscr{E} \subseteq \mathscr{P}(J)$ defined by: $X \in \mathscr{E}$ if and only if $\cup I_{j} \in \mathscr{D}$ is called the $\varrho$-image of $\mathscr{D}$ and is denoted by $\mathscr{D} / \varrho$. It is easy to see that $\bigcup_{j \in X} I_{j} \in \mathscr{D}$ is called the $Q$-image of $\mathscr{E}$ is a filter on $J$. Let $X \subseteq I$, we say that $X$ is $\varrho$-composable if there is $Y \subseteq J$ such that $X=\bigcup_{\mathcal{I}} l_{j}$. Let $\mathscr{G}$ be a filter on $I \times I$, then the family of all $\varrho$-composable sets for JaY $\varrho \in \mathscr{G}$ we call the family of $\mathscr{G}$-composable sets. This family coincides with $2^{I} \mid \mathscr{G}$. 